\title{
Perturbative algebraic QFT as a universal framework for constructing physically motivated models in quantum field theory
}

\author{
Kasia Rejzner* \\ University of York \\ E-mail: kasia.rejzner@york.ac.uk
}

\begin{abstract}
Perturbative algebraic quantum field theory is a formalism which allows to put perturbative QFT on a solid mathematical basis and solves many conceptual problems. It has proven to be a very successful framework for QFT on curved spacetimes, since it allows to separate the algebraic structure of the theory from the construction of a state. The main idea, inspired by the HaagKastler axiomatic framework, is to define a model of a QFT by giving a net of unital *-algebras, assigned to regions of spacetime. To construct such a model, one starts with a free classical theory, then obtains the free quantum theory via deformation quantization and finally introduces the interaction by means of Epstein-Glaser renormalization. In this overview talk I will show how this method works for the example of the scalar field and how this is related to Schwinger-Dyson equations.
\end{abstract}

Frontiers of Fundamental Physics 14 - FFP14,

15-18 July 2014

Aix Marseille University (AMU) Saint-Charles Campus, Marseille

\footnotetext{
${ }^{*}$ Speaker.
} 
To start the construction of a QFT model one needs the following input from physics:

- Spacetime M: a smooth globally hyperbolic (has a Cauchy surface) Lorentzian manifold. Such manifolds are necessarily non-compact.

- Configuration space $\mathcal{E}(M)$. This specifies the choice of objects one wants to study in the particular model (scalars, vectors, tensors, ...). Typically this is a space of smooth sections of some vector bundle $E \stackrel{\pi}{\rightarrow} M$ over $M$. For the scalar field, the configuration space is $\mathcal{E}(M) \equiv \mathcal{C}^{\infty}(M, \mathbb{R})$. More generally, one can choose $\mathcal{E}(M)$ as an infinite dimensional manifold modeled on a locally convex topological vector space. This generalization allows to treat theories like gravity or sigma models. Here I will focus on examples where the manifold $\mathcal{E}(M)$ is affine.

- Classical observables are modeled as smooth functionals on the configuration space $\mathcal{E}(M)$, i.e. elements of $\mathcal{C}^{\infty}(\mathcal{E}(M), \mathbb{R})$. One is primarily interested in certain classes of functionals relevant in physics. Let me first define the spacetime support of $F \in \mathcal{C}^{\infty}(\mathcal{E}(M), \mathbb{R})$ :

$\operatorname{supp} F=\{x \in M \mid \forall$ neigh, $U$ of $x \exists \varphi, \psi \in \mathcal{E}(M)$, supp $\psi \subset U$ such that $F(\varphi+\psi) \neq F(\varphi)\}$.

The support of $F$ essentially characterizes the region of spacetime that can thought of as the localization region of the classical observable corresponding to $F$. I will restrict myself to functionals that are compactly supported. A particularly important class of compactly supported smooth functionals consist of local ones. One says that $F \in \mathrm{C}^{\infty}(\mathcal{E}(M), \mathbb{R})$ is local if it is of the form: $F(\varphi)=\int_{M} f\left(j_{x}(\varphi)\right) d \mu(x)$, where $f$ is a function on the jet bundle over $M$ and $j_{x}(\varphi)=(\varphi(x), \partial \varphi(x), \ldots)$ is the jet of $\varphi$ at the point $x$. Let $\mathcal{F}_{\text {loc }}(M)$ denote the space of local functionals. In classical theory it is enough to consider functionals that are multilocal, i.e. they are sums of products of local functionals. The space of such functionals is denoted by $\mathcal{F}(M)$ and one can think of them as polynomials.

- Dynamics. To introduce the dynamics, I will use a modification of the Lagrangian formalism. Since the manifold $M$ is non-compact, I need to introduce a cutoff function into the Lagrangian. For the free scalar field the Lagrangian with a cutoff $f \in \mathcal{D}(M) \doteq \mathcal{C}_{c}^{\infty}(M, \mathbb{R})$ is given by

$$
L_{M}(f)(\varphi)=\frac{1}{2} \int\left(\nabla_{\mu} \varphi \nabla^{\mu} \varphi-m^{2} \varphi^{2}\right)(x) f(x) d \mu(x) .
$$

In general, a Lagrangian in the pAQFT framework is a family of maps (labeled by spacetimes) $L_{M}: \mathcal{D}(M) \rightarrow \mathcal{C}^{\infty}(\mathcal{E}(M), \mathbb{R})$, satisfying in addition:

1. $L_{M}(f+g+h)=L_{M}(f+g)-L_{M}(g)+L_{M}(g+h) ; f, g, h \in \mathcal{D}(M), \operatorname{supp} f \cap \operatorname{supp} h=\varnothing$.

2. $\operatorname{supp}\left(L_{M}(f)\right) \subseteq \operatorname{supp}(f)$.

Actions $S$ are equivalence classes of Lagrangians under the equivalence relation $L_{1} \sim L_{2}$ if $\operatorname{supp}\left(L_{1}-\right.$ $\left.L_{2}\right)(f) \subset \operatorname{supp} d f$. Physically, this amounts to identifying the Lagrangian densities that differ by a total derivative. The reason for identifying such Lagrangian is that they result in the same equations of motion (EOM's), i.e. they implement the same dynamics. In the pAQFT framework, the equations of motion are obtained from the Euler-Lagrange derivative of an action $S_{M}$, which is a map $S_{M}^{\prime}: \mathcal{E}(M) \rightarrow \mathcal{E}_{c}^{\prime}(M)$ defined as $\left\langle S_{M}^{\prime}(\varphi), h\right\rangle=\left\langle L_{M}(f)^{(1)}(\varphi), h\right\rangle$, where $f \equiv 1$ on supp $h$ (see the first diagram below) and $\varepsilon_{c}(M)$ denotes the space of compactly supported configurations. 

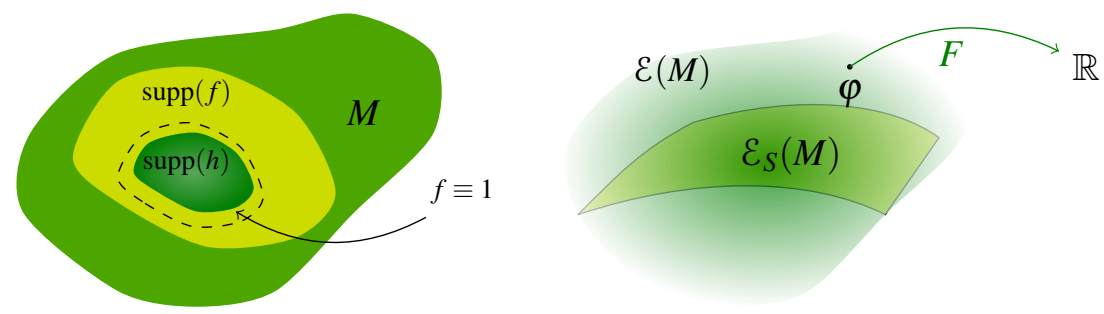

The EOM is the condition

$$
S_{M}^{\prime}(\varphi) \equiv 0 .
$$

Note that $S_{M}^{\prime}$ is a one-form on $\mathcal{E}(M)$ (an element of $\Gamma\left(T^{*} \mathcal{E}(M)\right)$ ), and $\varepsilon_{S}(M)$, the space of solutions of the EOM, is the zero locus of this form. Let me remark briefly on the manifold structure of $\mathcal{E}(M)$. I consider here only the affine case with the charts defined by

$$
\varphi+\mathcal{E}_{c}(M) \rightarrow \mathcal{E}_{c}(M), \varphi+\vec{\varphi} \mapsto \vec{\varphi}, \text { with } \varphi \in \mathcal{E}(M),
$$

where $\mathcal{E}_{c}(M)$ is equipped with its natural compact open topology, so $\mathcal{E}(M)$ is an infinite dimensional manifold modeled on $\mathcal{E}_{c}(M)$ (see [6] for details). In this setting $T^{*} \mathcal{E}(M)=\mathcal{E}(M) \times \mathcal{E}_{c}^{\prime}(M)$ (with the dual understood as the strong dual), so $\Gamma\left(T^{*} \mathcal{E}(M)\right) \cong \mathcal{C}^{\infty}\left(\mathcal{E}(M), \mathcal{E}_{c}^{\prime}(M)\right.$.

One calls elements of $\mathcal{E}_{S}(M)$ on-shell configurations. The second diagram above presents schematically $\mathcal{E}_{S}(M)$ as a subspace (in fact a submanifold) of $\mathcal{E}(M)$. One is then interested in the space $\mathcal{F}_{S}(M)$ of multilocal functionals on $\mathcal{E}_{S}(M)$. $\mathcal{F}_{S}(M)$ is called the space of classical onshell observables. It has a nice homological interpretation, which I will now present. As the first step, note that one can characterize $\mathcal{F}_{S}(M)$ as the quotient $\mathcal{F}(M) / \mathcal{F}_{0}(M)$, where $\mathcal{F}_{0}(M)$ is the ideal of $\mathcal{F}(M)$ consisting of elements that vanish on $\mathcal{E}_{S}(M)$. In "nice" cases, $\mathcal{F}_{0}(M)$ is generated by functionals of the form $\left\langle S_{M}^{\prime}, X\right\rangle$, where $X$ is a vector field on $\mathcal{E}(M)$, i.e. $X \in \Gamma(T \mathcal{E}(M)) \cong$ $\mathcal{C}^{\infty}\left(\mathcal{E}(M), \mathcal{E}_{c}(M)\right)$. For $\left\langle S_{M}^{\prime}, X\right\rangle$ to be multilocal, I need to restrict myself to vector fields $X$ which are in fact derivations of $\mathcal{F}(M)$. One calls such vector fields multilocal and the space consisting of such objects is denoted by $\mathcal{V}(M)$. I obtain a sequence:

$$
0 \rightarrow \operatorname{Ker}(\delta) \hookrightarrow \mathcal{V}(M) \stackrel{\delta}{\rightarrow} \mathcal{F}(M) \rightarrow 0,
$$

hence $\mathcal{F}_{S}(M)=H_{0}(\delta)$. In order to prove that $\mathcal{F}_{0}(M)$ is indeed equal to $\delta(\mathcal{V}(M))$, one has to examine the space of solutions $\mathcal{E}_{S}(M)$ in more detail. In particular, if $S$ is quadratic and $S_{M}^{\prime}$ is a normally hyperbolic operator, the relevant result was proven in [5]. For higher order $S$, the EOM's become non-linear and it can happen that they develop singularities, after a finite time, starting from smooth Cauchy data. In such cases, the space of smooth solutions $\mathcal{E}_{S}(M)$ could turn out to be very small and hard to control. In such situations it is better to take a more pragmatic point of view and define $\mathcal{F}_{S}(M)=\mathcal{F}(M) / \delta(\mathcal{V}(M))$. This way of thinking about on-shell observables agrees with the spirit of algebraic geometry and is more convenient from the point of view of quantization, so I adopt it also in this review.

In the next step I extend the map $\delta$ from $\mathcal{V}(M)$ to $\Lambda \mathcal{V}(M)$, the space of multivector fields defined as the exterior algebra (over $\mathcal{F}(M)$ ) of $\mathcal{V}(M)$, by requiring the graded Leibniz rule. This way I obtain a differential complex

$$
\ldots \rightarrow \Lambda^{2} \mathcal{V}(M) \stackrel{\delta}{\rightarrow} \mathcal{V}(M) \stackrel{\delta}{\rightarrow} \mathcal{F}(M) \rightarrow 0,
$$


called the Koszul complex. The kernel of $\delta$ in degree 1 consists of vector fields $X \in \mathcal{V}$, which satisfy $\left\langle S_{M}^{\prime}, X\right\rangle \equiv 0$, i.e. $\partial_{X} S_{M} \equiv 0$. These vector fields characterize directions in the configuration space $\mathcal{E}(M)$ in which the action $S$ is constant; one calls these vector fields local symmetries. The space of symmetries includes, in particular, elements of the form $\delta\left(\Lambda^{2} \mathcal{V}(M)\right)$, where $\Lambda^{2} \mathcal{V}(M)$ is the second exterior power of $\mathcal{V}$. Such symmetries are called trivial. It is now clear that $H_{1}(\Lambda \mathcal{V}(M), \delta)$ characterizes in fact the space of non-trivial local symmetries. In the simplest case (e.g. real scalar field), $S$ doesn't have non-trivial local symmetries and the Koszul complex is a resolution of $\mathcal{F}_{S}(M)$, i.e. $H_{0}(\Lambda \mathcal{V}(M), \delta)=\mathcal{F}_{S}(M)$ and $H_{k}(\Lambda \mathcal{V}(M), \delta)$ for $k \neq 0$.

Note that $\Lambda \mathcal{V}(M)$, as the space of multivector fields on $\mathcal{E}(M)$, is equipped with natural graded bracket, the Schouten bracket: $\{X, F\}=\partial_{X} F$ for $X$ a vector field and $F$ a function; $\{X, Y\}=[X, Y]$ for $X, Y$ a vector fields; $\{.,$.$\} is extended to higher orders by the graded Leibniz rule. One can$ check that $\{.,$.$\} defined this way is of degree -1$ and it satisfies the graded Jacobi identity. The structure $(\Lambda \mathcal{V}(M),\{.,\}$.$) is an example of a Gerstenhaber algebra and together with \delta$ one obtains a differential Gerstenhaber algebra.

I will now focus on the example of the scalar field and show how it can be quantized within the pAQFT framework. I start with the free theory described by the Lagrangian (1) and after quantizing it I will introduce the interaction in a perturbative way. The equation of motion is $S_{M}^{\prime}(\varphi)=P \varphi=0$, where $P=\square+m^{2}$ is the Klein-Gordon operator. Since $M$ is assumed to be globally hyperbolic, $P$ posses the retarded and advanced Green's functions $\Delta^{R}, \Delta^{A}$. They satisfy $P \circ \Delta^{R / A}=\mathrm{id}_{\mathcal{D}(M)}$ and $\Delta^{R / A} \circ\left(\left.P\right|_{\mathcal{D}(M)}\right)=\mathrm{id}_{\mathcal{D}(M)}$ as well as the support properties: $\operatorname{supp}\left(\Delta^{R}\right) \subset\left\{(x, y) \in M^{2} \mid y \in\left(\bar{V}_{-}\right)_{x}\right\}$ and $\operatorname{supp}\left(\Delta^{A}\right) \subset\left\{(x, y) \in M^{2} \mid y \in\left(\bar{V}_{+}\right)_{x}\right\}$, where $\bar{V}_{ \pm}$is the closed future/past lightcone. Their difference is the causal propagator $\Delta \doteq \Delta^{R}-\Delta^{A}$. The singularities of the distribution $\Delta$ are well understood, as one can explicitly determine its WF set

$$
\mathrm{WF}(\Delta)=\left\{\left(x, k ; x^{\prime},-k^{\prime}\right) \in \dot{T}^{*} M^{2} \mid(x, k) \sim\left(x^{\prime}, k^{\prime}\right)\right\},
$$

where $\sim$ means that there is a null geodesic connecting $x$ and $x^{\prime}$ and $k^{\prime}$ is the parallel transport of $k$ along it. Note that $\operatorname{WF}(\Delta)$ breaks down into two disjoint components, one with $k$ on the future lightcone and the other with $k$ on the past lightcone. Therefore, one can decompose $\Delta$ as $\frac{i}{2} \Delta=\Delta_{+}-H$, where $\Delta_{+}$is the positive frequency part, i.e.:

$$
\mathrm{WF}\left(\Delta_{+}\right)=\left\{\left(x, k ; x^{\prime},-k^{\prime}\right) \in \dot{T} M^{2} \mid(x, k) \sim\left(x^{\prime}, k^{\prime}\right), k \in\left(\bar{V}_{+}\right)_{x}\right\} .
$$

One requires $H$ to be a symmetric bi-solution for $P$. Moreover, $\Delta_{+}$is required to be positive definite. On the Minkowski spacetime, $\Delta_{+}$is just the Wightman 2-point function. On general globally hyperbolic spacetimes, the choice of $\Delta_{+}$is non-canonical and this ambiguity corresponds to the non-existence of a preferred vacuum state (see for example [3] for a detailed discussion of these issues). The Feynman propagator is now defined by:

$$
\Delta_{F}=\frac{1}{2}\left(\Delta^{A}+\Delta^{R}\right)+H .
$$

Properties of the WF set of $\Delta_{+}$motivate to define the space of microcausal functionals $\mathcal{F}_{\mu \mathrm{c}}(M)$ as the space consisting of functionals $F$ with

$$
\mathrm{WF}\left(F^{(n)}(\varphi)\right) \subset \Xi_{n}, \quad \forall n \in \mathbb{N}, \forall \varphi \in \mathfrak{E}(M),
$$


where $\Xi_{n} \doteq T^{*} M^{n} \backslash\left\{\left(x_{1}, \ldots, x_{n}, k_{1}, \ldots k_{n}\right) \mid k_{i} \in\left(\bar{V}_{+}\right)_{x_{i}} \cup\left(\bar{V}_{-}\right)_{x_{i}}, i=1 \ldots n\right\}$. For $F, G \in \mathcal{F}_{\mu \mathrm{c}}(M)$, I define now the $\star$-product (deformation of the pointwise product) as:

$$
(F \star G)(\varphi) \doteq \sum_{n=0}^{\infty} \frac{\hbar^{n}}{n !}\left\langle F^{(n)}(\varphi),\left(\Delta_{+}\right)^{\otimes n} G^{(n)}(\varphi)\right\rangle
$$

The free QFT is defined as $\mathfrak{A}_{0}(M) \doteq\left(\mathcal{F}_{\mu \mathrm{c}}(M), \star\right)$.

The interaction is introduced by means of time-ordered products. I start with defining these on a particularly well-behaving class of functionals and later on I will explain how to extend this definition to a larger class. The space of regular functionals $\mathcal{F}_{\text {reg }}(M)$ is defined as the space of functionals whose derivatives are test functions, i.e. $F^{(n)}(\varphi) \in \mathcal{D}\left(M^{n}\right)$. In a similar way we define also the space of regular vector fields $\mathcal{V}_{\text {reg }}(M)$. The time-ordering operator $\mathcal{T}$ is defined on $\mathcal{F}_{\text {reg }}(M)$ as:

$$
\mathcal{T} F(\varphi) \doteq \sum_{n=0}^{\infty} \frac{\hbar^{n}}{n !}\left\langle F^{(2 n)}(\varphi),\left(i \Delta_{F}\right)^{\otimes n}\right\rangle
$$

and the time-ordered product $\cdot \mathcal{T}$ on $\mathcal{T}\left(\mathcal{F}_{\text {reg }}(M)[[\hbar]]\right)$ is given by

$$
F \cdot{ }_{\mathcal{T}} G \doteq \mathcal{T}\left(\mathcal{T}^{-1} F \cdot \mathcal{T}^{-1} G\right)
$$

I now have an algebraic structure with two products $\left(\mathcal{F}_{\text {reg }}(M)[[\hbar]], \star, \cdot \mathcal{T}\right)$, where $\star$ is non-commutative, $\cdot_{\mathcal{T}}$ is commutative and they are related by a causal relation $F \cdot{ }_{\mathcal{T}} G=F \star G$, if the support of $F$ is later than the support of $G$. By interaction term I mean functional $V$ (for a moment I assume that it belongs to $\mathcal{F}_{\text {reg }}(M)$ ) and using the commutative product $\cdot_{\mathcal{T}}$ I define the $S$-matrix corresponding to this interaction as:

$$
\mathcal{S}(V) \doteq e_{\mathcal{T}}^{V}=\mathcal{T}\left(e^{\mathcal{T}^{-1} V}\right)
$$

Interacting fields are defined by the formula of Bogoliubov:

$$
R_{V}(F) \doteq\left(e_{\mathcal{T}}^{V}\right)^{\star-1} \star\left(e_{\mathcal{T}}^{V} \cdot \mathcal{T} F\right)
$$

Let me now consider what happens with the Gerstenhaber algebra introduced earlier. Explicit calculation shows that

$$
\delta_{\mathcal{T}}:=\mathcal{T}^{-1} \circ \delta \circ \mathcal{T}=\delta+i \hbar \triangle,
$$

where $\triangle$ acts on vector fields $X \in \mathcal{V}_{\text {reg }}(M)$ as $\triangle X(\varphi)=\int \frac{\delta X_{x}}{\delta \varphi(x)}(\varphi)$ with $X(\varphi)=\int X_{x}(\varphi) \frac{\delta}{\delta \varphi(x)}$. It is remarkable that the operator $\triangle$ is "almost" a derivation of $\Lambda V_{\text {reg }}(M)$ and the failure is characterized by $\{.,$.$\} , i.e:$

$$
\triangle(X \wedge Y)-\triangle(X) \wedge Y-(-1)^{|X|} X \wedge \triangle(Y)=\{X, Y\},
$$

where $|X|$ is the degree of the multivector field $X$. The triple $\left(\Lambda \mathcal{V}_{\text {reg }}(M),\{.,\},. \triangle\right)$ is an example of a mathematical structure called the $B V$ algebra. The BV structure is a universal feature of $\mathrm{pAQFT}$ models, although it was first discovered in physics [1] in the context of gauge theories. For the free scalar field the relation between $\delta_{\mathcal{T}}$ and $\delta$ corresponds to the Schwinger-Dyson equation.

$$
\left(\delta_{\mathcal{T}} X\right)(\varphi)=\int\left(X_{x} \cdot \mathcal{T} \frac{\delta S}{\delta \varphi(x)}\right)(\varphi)=\int X_{x}(\varphi) \frac{\delta S(\varphi)}{\delta \varphi(x)}-i \hbar \int \frac{\delta X_{x}}{\delta \varphi(x)}(\varphi)
$$


Note that $\int X_{x}(\varphi) \frac{\delta S}{\delta \varphi(x)}=\int\left(X_{x} \star \frac{\delta S}{\delta \varphi(x)}\right)(\varphi)$. Hence, $\int\left(X_{x} \cdot \mathcal{T} \frac{\delta S}{\delta \varphi(x)}\right)=-i \hbar \int \frac{\delta X_{x}}{\delta \varphi(x)}$ modulo the $\star-$ ideal generated by EOM's (this is the algebraic Schwinger-Dyson equation).

Up to now, I have considered only the regular functionals $\mathcal{F}_{\text {reg }}(M)$. Now I want to generalize all the constructions also to the local functionals. Because of the WF set properties of $\Delta_{F}$, the timeordered product $\cdot \mathcal{T}$ is not well defined on local, non-linear functionals, but the physical interaction is usually local! The renormalization problem is therefore to extend $\mathcal{S}($.$) to local arguments. This$ is reduced to extending the $n$-fold time-ordered products, since

$$
\mathcal{S}(V)=\sum_{n=0}^{\infty} \frac{1}{n !} \mathcal{T}_{n}(V, \ldots, V) .
$$

The time-ordered product $\mathcal{T}_{n}\left(F_{1}, \ldots, F_{n}\right) \doteq F_{1} \cdot \mathcal{\tau} \ldots \cdot{ }_{\mathcal{\tau}} F_{n}$ of $n$ local functionals is well defined if their supports are pairwise disjoint. To extend $\mathcal{T}_{n}$ to arbitrary local functionals I follow the causal approach of Epstein and Glaser (causal perturbation theory), where one proves the existence of the renormalized $n$-fold time ordered products $\mathcal{T}_{n}^{\mathrm{r}}$ such that $\mathcal{T}_{0}^{\mathrm{r}}=0, \mathcal{T}_{1}^{\mathrm{r}}=\mathrm{id} ; \operatorname{supp} \mathcal{T}_{n}^{\mathrm{r}}\left(F_{1}, \ldots, F_{n}\right) \subset$ $\cup \operatorname{supp} F_{i}$ and the Causal factorization property holds. The latter means that, if the supports of $F_{1}, \ldots, F_{i}$ are later than the supports of $F_{i+1}, \ldots, F_{n}$, then

$$
\mathcal{T}_{n}^{\mathrm{r}}\left(F_{1} \otimes \cdots \otimes F_{n}\right)=\mathcal{T}_{i}^{\mathrm{r}}\left(F_{1} \otimes \cdots \otimes F_{i}\right) \star \mathcal{T}_{n-i}^{\mathrm{r}}\left(F_{i+1} \otimes \cdots \otimes F_{n}\right) .
$$

By the theorem of Epstein and Glaser I know that the extension exists, but is not unique. Furthermore, in [4] it was shown that the renormalized time-ordered product $\cdot_{\mathcal{\tau}}$ is an associative product on $\mathcal{T}^{\mathrm{r}}(\mathcal{F}(M))$ given by

$$
F \cdot \mathcal{T}^{\mathrm{r}} G \doteq \mathcal{T}^{\mathrm{r}}\left(\mathcal{T}^{\mathrm{r}-1} F \cdot \mathcal{T}^{\mathrm{r}-1} G\right),
$$

where $\mathcal{T}^{\mathrm{r}}: \mathcal{F}(M)[[\hbar]] \rightarrow \mathcal{T}^{\mathrm{r}}(\mathcal{F}(M))[[\hbar]]$ is defined as $\mathcal{T}^{\mathrm{r}}=\left(\bigoplus_{n} \mathcal{T}_{n}^{\mathrm{r}}\right) \circ \beta$ and $\beta: \mathcal{F}(M) \rightarrow S^{\bullet} \mathcal{F}_{\text {loc }}^{(0)}(M)$ is the inverse of multiplication $m$ and $\mathcal{T}_{n}^{\mathrm{r}}$ is a family of renormalized time-ordered products. This result, together with the anomalous Master Ward Identity proven in [2], allows one to write the renormalized Schwinger-Dyson equation as

$$
\delta_{\mathcal{T}^{r}}\left(e_{\mathcal{T}^{\mathrm{r}}}^{i X / \hbar}\right)=e_{\mathcal{T}^{\mathrm{r}}}^{i X / \hbar} \cdot \mathcal{T}^{\mathrm{T}}\left(\delta X+\frac{1}{2}\{X, X\}-i \hbar \triangle^{\mathrm{r}}(X)\right),
$$

where $\triangle^{\mathrm{r}}$ is the renormalized BV Laplacian, obtained recursively from the formula for the anomaly term provided in [2].

\section{References}

[1] I.A. Batalin, G.A. Vilkovisky, Gauge Algebra And Quantization, Phys. Lett. 102B (1981) 27.

[2] F. Brennecke, M. Dütsch, Removal of violations of the Master Ward Identity in perturbative QFT, Rev.Math.Phys. 20 (2008) 119-172.

[3] R. Brunetti, K. Fredenhagen, R. Verch, The generally covariant locality principle - A new paradigm for local quantum field theory, Commun. Math. Phys. 237 (2003) 31-68.

[4] K. Fredenhagen, K. Rejzner, Batalin-Vilkovisky formalism in perturbative algebraic quantum field theory, Commun. Math. Phys. 317 (2013), 697-725.

[5] K. Fredenhagen, K. Rejzner, "QFT on curved spacetimes: axiomatic framework and examples," [math-ph/1412.5125].

[6] A. Kriegl, P. Michor, Convenient setting of global analysis, Mathematical Surveys and Monographs 53, American Mathematical Society, Providence 1997. 